\title{
Research article \\ Assessment of carotid artery intima media thickness in prehypertension
}

\author{
Anahita R. Shenoy Basti ${ }^{1}$, Shrilakshmi M. Prabhu' ${ }^{2}$, Ram Shenoy Basti ${ }^{3}$ \\ ${ }^{1}$ Associate Professor, Department of Physiology, ${ }^{2}$ Assistant Professor, Department of Medicine, ${ }^{3}$ Professor, Department of \\ Radiology, Father Muller Medical College, Mangalore 575002, Karnataka India
}

(Received: August $2020 \quad$ Revised: February $2021 \quad$ Accepted: March 2021)

Corresponding author: Anahita R. Shenoy Basti. Email: anahitakini@yahoo.in

\begin{abstract}
Introduction and Aim: Increased carotid artery intima media thickness (CIMT) has been linked to the development of cardiovascular disease (CVD). CVD is a major cause of mortality and morbidity in India. Increased CIMT has been documented in Hypertension (HT), thus putting them at a higher risk of CVD. Early identification and awareness of risk factors leading to increased morbidity and mortality could help in reducing its incidence. The aim of the study was to measure CIMT in prehypertensive Indian population.
\end{abstract}

Materials and Methods: In this analytical observational study, common carotid artery IMT was measured using B mode ultrasonography in 46 individuals having blood pressure in the prehypertensive range and 46 individuals with normal blood pressure. Cardiovascular risk factors like body mass index (BMI), fasting blood glucose (FBS), and lipid profile, which are known to influence CIMT, were also assessed and compared between the two groups.

Results: CIMT was significantly higher in prehypertensives as compared to subjects having normal blood pressure. BMI, FBS, and lipid profile was found to be comparable between the two groups.

Conclusions: The presence of increased CIMT in prehypertensives as compared to controls indicates an increased risk of adverse cardiovascular events.

Keywords: Prehypertension; carotid artery intima media thickness; atherosclerosis; blood pressure.

\section{INTRODUCTION}

$\mathrm{C}$ arotid intima medial thickness (CIMT) is a measure of the combined thickness of the intima and media layers of the carotid artery. It is a non-invasive, reproducible and inexpensive tool that can be used to identify target organ damage. Increased CIMT is due to factors that cause the development and progression of atherosclerosis (1). CIMT has been reported as a marker of subclinical and asymptomatic atherosclerotic vascular diseases. CIMT is raised in hypertensive subjects which puts them at a greater risk of adverse cardiovascular events (2). Measurement of CIMT in subjects with BP in the prehypertensive range has shown varied results, with some studies reporting an increase in CIMT as compared to normotensives while another showing no significant difference (3-5).

The term Prehypertension was first introduced in "The Seventh Report of the Joint National Committee on the Prevention, Detection, Evaluation, and Treatment of High Blood Pressure" (JNC 7) in the year 2003. It is defined as the presence of systolic blood pressure (SBP) between $120 \mathrm{mmHg}$ to $139 \mathrm{mmHg}$ and/or a diastolic BP (DBP) between $80 \mathrm{mmHg}$ and $89 \mathrm{mmHg}$ (6). Prevalence of prehypertension in the urban south Indian population is found to be high, with some studies reporting it to be as high as $55 \%$ (7). Prehypertension holds clinical relevance as it is seen to be a precursor to hypertension (HT) and is also associated with increased morbidity and mortality from cardiovascular disease (CVD) $(8,9)$. However, not all studies have shown a significant association; with a 12 year follow up cohort study not finding prehypertension to be independently associated with increased all-cause or CVD mortality after adjustment for age, gender, race, and other CVD risk factors (10).

Thus, in this study, we aim to measure CIMT in prehypertensives. We also evaluated other cardiovascular risk factors like body mass index (BMI), Fasting blood sugar (FBS) and Lipid profile in prehypertensives.

\section{MATERIALS AND METHODS}

This analytical observational study was initiated after approval from the institutional ethical committee. Patients attending medicine OPD, health check-up patients, as well as inpatient were screened to identify subjects with $\mathrm{BP}$ in the prehypertensive range (SBP $120-139 \mathrm{~mm}$ of $\mathrm{Hg}$ and/or DBP- $80-89 \mathrm{~mm} \mathrm{Hg}$ ). These formed the cases. Age and sex-matched subjects with normal $\mathrm{BP}(\mathrm{SBP}<120 \mathrm{~mm}$ of $\mathrm{Hg}, \mathrm{DBP}<80 \mathrm{~mm}$ of $\mathrm{Hg})$ formed the control group. Subjects with hypertension or previously on antihypertensive drugs, coronary artery diseases, on lipid-lowering drugs, diabetes and having any renal pathology were excluded. The subjects were explained about the study in their own language and written informed consent was taken. A total of 46 subjects were inducted into both groups. The sample size of 46 was determined using, $95 \%$ confidence interval and $90 \%$ power (3).

These subjects underwent detailed clinical examinations, biochemical investigations and CIMT 
thickness assessment. Clinical examination consisted of history taking for the presence of risk factors for CVD, medications taken and presence of any symptoms suggestive of CVD.

Anthropometric examinations to determine body mass index (BMI) were conducted. Height (ht) was measured using a standard stadiometer with the subjects standing in an erect posture. The readings were taken to the nearest $0.1 \mathrm{~cm}$. Weight (wt) was measured using a calibrated weighing machine. The BMI was calculated using the formula, wt $(\mathrm{kg}) / \mathrm{ht}\left(\mathrm{m}^{2}\right)$.

This was followed by systemic examinations and measurement of blood pressure. After a rest period of 5 minutes, BP recordings were taken till 2 readings were within $5 \mathrm{~mm}$ of $\mathrm{Hg}$ of each other. BP was measured in the right arm in sitting position, using a mercury sphygmomanometer. Average of two BP recordings were considered for the study.

Under aseptic precautions, venous blood was drawn to estimate FBS and lipid profile. FBS was estimated using the hexokinase method. Serum total cholesterol was estimated by cholesterol oxidase/peroxidase method, serum triglyceride (TG) by enzymatic kinetic and glycerol phosphate method, serum HDL cholesterol by direct method polymer/detergent, serum total cholesterol/HDL cholesterol ratio by calculation and serum VLDL by calculation from TG values.

Common carotid artery intima media thickness was evaluated bilaterally in the distal segment of the artery using B mode ultrasound with high-frequency linear transducer by a skilled radiologist who was unaware of the clinical status of the subject (blinded). The subject was made to lie down in supine position, the neck was extended by placing it above a pillow and head was turned to the opposite side of the recording. The CIMT was taken as the distance between the leading edge of the lumen-intima interface and the leading edge of the media-adventitia interface of the distal common carotid artery. CIMT was measured on both sides. Philips Affiniti 70 machine with e L 18-4 linear probe was used to measure the CIMT.

The study subjects were further divided into subgroups - elevated blood pressure (SBP 120-129mm of $\mathrm{Hg}$ and DBP $<80 \mathrm{~mm}$ of $\mathrm{Hg}$ ) and stage I HT (SBP130-139mm of $\mathrm{Hg}$ or DBP $80-89 \mathrm{~mm}$ of $\mathrm{Hg}$ ).

Mean, standard deviation, ' $t$ ' test and chi-square test were used to compare the data between the two groups. Bonferroni test was used to compare CIMT between the elevated BP subgroups and controls, between subgroup stage I HT and controls.

\section{RESULTS}

We have compared CIMT in prehypertensives (cases) with age and sex-matched subjects with normal BP (control). Table 1 compares the age, gender and family history of diabetes mellitus (DM) and HT between the two groups. The groups were comparable on these parameters.

Table 2 Compares the BMI, BP, FBS, lipid profile and CIMT between the two study groups. The mean systolic blood pressure (SBP) in cases was found to be $127.09 \pm 6.11 \mathrm{~mm}$ of $\mathrm{Hg}$ and diastolic blood pressure (DBP) was $81.83 \pm 3.70 \mathrm{~mm}$ of $\mathrm{Hg}$. In controls, SBP and DBP were $113.43 \pm 4.69 \mathrm{~mm}$ of $\mathrm{Hg}$ and $73.87 \pm 4.22$ $\mathrm{mm}$ of $\mathrm{Hg}$ respectively. CIMT was found to be significantly higher in prehypertensives, BMI, FBS and lipid profile was comparable to the normotensives. p-value of $<0.05$ was considered significant.

Table 1: Comparison of age, sex, family history of HT and DM between cases and controls

\begin{tabular}{|c|c|c|c|c|c|}
\hline \multicolumn{2}{|c|}{ Parameters } & \multicolumn{2}{c|}{ Cases } & \multicolumn{2}{c|}{ Control } \\
\hline \multicolumn{2}{|c|}{ Age } & Count & Column N \% & Count & Column N \% \\
\hline & $20-30$ & 3 & 6.5 & 3 & 6.5 \\
\hline & $31-40$ & 8 & 17.4 & 16 & 34.8 \\
\hline & $41-50$ & 10 & 21.7 & 14 & 30.4 \\
\hline & Above 50 & 25 & 54.3 & 13 & 28.3 \\
\hline & Total & 46 & 100.0 & 46 & 100.0 \\
\hline & Female & 19 & 41.3 & 22 & 47.8 \\
\hline & Male & 27 & 58.7 & 24 & 52.2 \\
\hline Family h/o HT & Total & 46 & 100.0 & 46 & 100.0 \\
\hline & Yes & 14 & 30.4 & 11 & 23.9 \\
\hline & No & 32 & 69.6 & 35 & 76.1 \\
\hline Family h/o DM & Total & 46 & 100.0 & 46 & 100.0 \\
\hline & Yes & 12 & 26.1 & 14 & 30.4 \\
\hline & No & 34 & 73.9 & 32 & 69.6 \\
\hline & Total & 46 & 100.0 & 46 & 100.0 \\
\hline
\end{tabular}


Table 2: BMI, BP, FBS, Lipid profile and CIMT between cases and controls. Values expressed in mean \pm standard deviation. $\mathrm{P}<0.05$ considered significant

\begin{tabular}{|c|c|c|c|}
\hline$(n=46)$ & Cases & Controls & P value \\
\hline BMI kg/m2 & $25.41 \pm 4.78$ & $24.71 \pm 3.45$ & 0.42 \\
\hline SBP $(\mathrm{mm}$ of $\mathrm{Hg})$ & $127.09 \pm 6.11$ & $113.43 \pm 4.69$ & $<0.001^{*}$ \\
\hline $\mathrm{DBP}(\mathrm{mm}$ of $\mathrm{Hg})$ & $81.83 \pm 3.70$ & $73.87 \pm 4.22$ & $<0.001^{*}$ \\
\hline FBS mg/dl & $100.63 \pm 7.16$ & $101.37 \pm 13.02$ & 0.73 \\
\hline S. Total cholesterol (mg/dl) & $198.30 \pm 40.00$ & $187.91 \pm 33.15$ & 0.17 \\
\hline S. LDL cholesterol (mg/dl) & $142.86 \pm 41.49$ & $134.56 \pm 32.58$ & 0.28 \\
\hline S. Triglycerides (mg/dl) & $134.00 \pm 57.74$ & $137.07 \pm 77.09$ & 0.83 \\
\hline S. HDL cholesterol $(\mathrm{mg} / \mathrm{dl})$ & $47.00 \pm 14.03$ & $44.43 \pm 11.48$ & 0.34 \\
\hline Serum total cholesterol /HDL ratio & $4.62 \pm 1.80$ & $4.48 \pm 1.26$ & 0.66 \\
\hline Serum VLDL $(\mathrm{mg} / \mathrm{dl})$ & $26.37 \pm 13.22$ & $25.57 \pm 13.49$ & 0.77 \\
\hline CIMT (mm) right common carotid artery & $0.54 \pm 0.16$ & $0.45 \pm 0.12$ & $0.004 *$ \\
\hline CIMT (mm) left common carotid artery & $0.58 \pm 0.22$ & $0.45 \pm 0.12$ & $<0.001^{*}$ \\
\hline$(n=46)$ & Cases & Controls & P value \\
\hline $\mathrm{BMI} \mathrm{kg} / \mathrm{m} 2$ & $25.41 \pm 4.78$ & $24.71 \pm 3.45$ & 0.42 \\
\hline SBP $(\mathrm{mm}$ of $\mathrm{Hg})$ & $127.09 \pm 6.11$ & $113.43 \pm 4.69$ & $<0.001^{*}$ \\
\hline DBP $(\mathrm{mm}$ of $\mathrm{Hg})$ & $81.83 \pm 3.70$ & $73.87 \pm 4.22$ & $<0.001^{*}$ \\
\hline FBS mg/dl & $100.63 \pm 7.16$ & $101.37 \pm 13.02$ & 0.73 \\
\hline S. Total cholesterol (mg/dl) & $198.30 \pm 40.00$ & $187.91 \pm 33.15$ & 0.17 \\
\hline S. LDL cholesterol (mg/dl) & $142.86 \pm 41.49$ & $134.56 \pm 32.58$ & 0.28 \\
\hline S. Triglycerides (mg/dl) & $134.00 \pm 57.74$ & $137.07 \pm 77.09$ & 0.83 \\
\hline S. HDL cholesterol $(\mathrm{mg} / \mathrm{dl})$ & $47.00 \pm 14.03$ & $44.43 \pm 11.48$ & 0.34 \\
\hline Serum total cholesterol /HDL ratio & $4.62 \pm 1.80$ & $4.48 \pm 1.26$ & 0.66 \\
\hline Serum VLDL (mg/dl) & $26.37 \pm 13.22$ & $25.57 \pm 13.49$ & 0.77 \\
\hline
\end{tabular}

*statistically significant

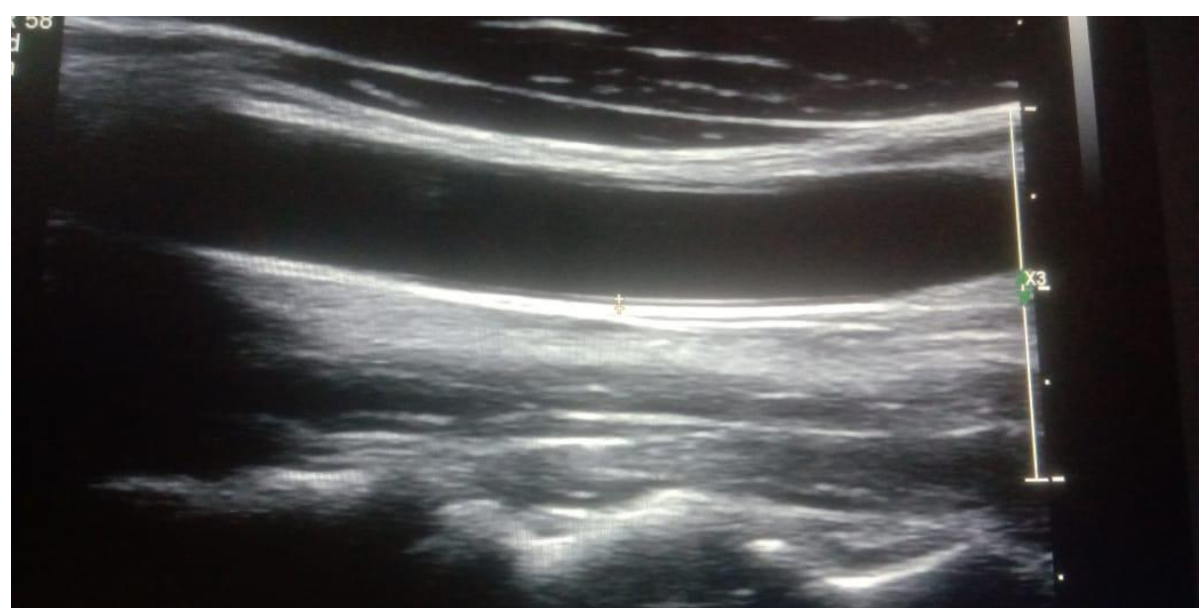

Fig. 1: Measurement CIMT of the common carotid artery using B mode ultrasonography

\section{DISCUSSION}

In our study, CIMT was found to be significantly higher in prehypertensives as compared to individuals with normal blood pressure. Other cardiovascular risk factors like BMI, FBS, and lipid profile were found to be comparable between the two groups. We further divided the subjects into subgroups -elevated blood pressure group, stage I HT and normal BP group(controls). CIMT was found to be comparable between elevated BP and controls (p-value- 1), a significantly higher CIMT was found in stage I HT when compared to controls ( $\mathrm{p}$-value $<0.001$ ).

Our findings were similar to a study by Manios et al., where prehypertensives had higher CIMT than normotensives even after adjusting for baseline characteristics (3). Another study on young asymptomatic men with SBP in the range of 130$140 \mathrm{~mm}$ of $\mathrm{Hg}$ or DBP $80-89 \mathrm{~mm}$ of $\mathrm{Hg}$ reported higher CIMT (4). CIMT was found to be higher in prehypertension across age groups, both in young and middle-aged individuals $(11,12)$. However, a study has reported unfavourable metabolic profilesignificantly higher body mass and waist circumference, higher TC, HDL- C and TG values in prehypertensives but CIMT in them was comparable to normotensive individuals, this is in contradiction to our findings (5). A $12 \mathrm{yr}$ cohort study also did not find prehypertension to be associated with increased allcause or CVD mortality after adjustment for age, gender, race, and other CVD risk factors (10).

Keeping these inconsistencies in mind we measured CIMT between age and sex- matched prehypertensives and normotensives. As values of 
CIMT are influenced by factors such as age, gender and ethnicity both groups in our study were matched for age and gender to ensure comparability (13).

CIMT was found to be $0.54 \pm 0.16 \mathrm{~mm}$ in the right common carotid artery and $0.58 \pm 0.22 \mathrm{~mm}$ in the left common carotid artery in prehypertensives and $0.45 \pm 0.12 \mathrm{~mm}$ right common carotid artery and $0.45 \pm 0.12 \mathrm{~mm}$ in the left common carotid artery in individuals with normal blood pressure. Average and maximum values of $0.67 \mathrm{~mm}$ and $0.70 \mathrm{~mm}$ have been reported in healthy Indian adults and values above $1 \mathrm{~mm}$ is known to be associated with a significant risk of coronary heart disease across age groups $(14,15)$. Although prehypertensives in our study had significantly higher CIMT as compared to normotensives, it was still within the normal range for the Indian population.

When we further classified the study subjects based on New American College of Cardiology/ American Heart Association (ACC/AHA) guidelines into elevated blood pressure (SBP 120-129mm of $\mathrm{Hg}$ and DBP $<80 \mathrm{~mm}$ of $\mathrm{Hg}$ ) and stage $1 \mathrm{HT}$ (SBP 130-139mm of $\mathrm{Hg}$ or DBP $80-89 \mathrm{~mm}$ of $\mathrm{Hg}$ ), significant difference in CIMT was found only between stage I HT and controls. ACC/AHA published their guidelines in 2017 for arterial HT where they have reduced the cut off for HT to 130 and/or $80 \mathrm{mmHg}$ however recommended antihypertensive medication remained the same in the general population (16). Reducing the cut off for diagnosis of HT but not for treatment could be a method to introduce regular follow up and lifestyle modifications which could help to reduce the incidence of HT and its complications. Thus, importance is now being laid on prevention of the disease rather than palliation.

We also evaluated other cardiovascular risk factors like BMI, FBS, lipid profile in prehypertensives, which was found to be comparable to the normotensives, and no significant changes were observed. Obesity, elevated blood sugar, unfavourable lipid profile act as confounding factors and are known to independently influence CIMT. Unfavourable BMI and significantly higher total cholesterol and triglyceride values in prehypertensives have been reported in other studies $(5,17)$. A study by Lankarani et al., CIMT was reported to be significantly higher in patients with higher BMI, higher waist circumference and higher triglyceride levels (18). As our study groups were comparable in these above parameters, it eliminated their confounding influence (TO REMOVE as it is a repetition).

Prehypertension is the interest of our study as it has a high prevalence in India. A prevalence of $32.3 \%$ was found in the upper socioeconomic north Indian population (19). Another study done in south Indian reported the prevalence to be $36.1 \%$, prevalence as high as $55 \%$ has also been reported $(20,7)$. It was observed that $23.4 \%$ of prehypertensives developed
HT at the end of a 2 year follow up in a study done in Kerala (8). Apart from developing HT, studies have found prehypertensives to be associated with left ventricular remodelling, diastolic dysfunction and also hypertrophy (21). Long term follow up of prehypertensive patients have found them to be at higher risk of cardiovascular disease and stroke (22). It was shown that for each $0.1 \mathrm{~mm}$ increase in common CIMT, an $11 \%$ increase in the risk of myocardial infarction was noticed (23).

A rapid increase in the prevalence of prehypertension and HT in India has necessitated the development of aggressive strategies for its prevention. Identifying atrisk individuals accurately is the cornerstone of preventive strategies. Measurement of CIMT could be used as a screening tool to identify at- risk individuals. Focusing the health care resources on these high-risk individuals to prevent the occurrence of HT is essential. Also, awareness of the risk for the development of HT could improve the patient's lifestyle and health-seeking behaviour.

\section{Limitations}

As our study design is cross-sectional, the cause and effect relationship between prehypertension and carotid artery intima media thickness could not be established.

\section{CONCLUSION}

We in our study have shown increased CIMT in prehypertensive individuals compared to normotensives. Increased CIMT is an indicator of target organ damage. Since there exists a controversy regarding the use of medication for the treatment of prehypertension, CIMT can be used as a tool for cardiovascular risk stratification and identifying atrisk individuals.

\section{ACKNOWLEDGEMENT}

We would like to acknowledge Fr. Muller Research Center for the grant to conduct the study.

\section{CONFLICT OF INTEREST}

Authors declare no conflict of interest.

\section{REFERENCES}

1. Stein, J. H., Korcarz, C. E., Hurst, R. T., Lonn, E., Kendall, C. B., Mohler, E. R., et al., Use of carotid ultrasound to identify subclinical vascular disease and evaluate cardiovascular disease risk: A consensus statement from the American Society of Echocardiography Carotid IntimaMedia Thickness Task Force. Endorsed by the Society for Vascular Medicine. J Am Soc Echocardiogr. 2008; 21: 93111.

2. Takase, H., Sugiura, T., Murai, S., Yamashita, S., Ohte, N., Dohi, Y. Carotid intima-media thickness is a novel predictor of new onset of hypertension in normotensive subjects. Medicine (Baltimore) 2017; 96(31): e7710.

3. Efstathios, M., Geogios, T., Eleni, K., Kinmon, S., Christos, P., Savas, T., et al., Impact of prehypertension on common carotid artery intima-media thickness and left ventricular mass. Stroke. 2009; 40: 1515-1518. 
4. Toikka, J. O., Laine, H., Ahotupa, M., Haapanen, A., Viikari, J. S., Hartiala, J. J., Raitakari, O. T., Increased arterial intimamedia thickness and in vivo LDL oxidation in young men with borderline hypertension. Hypertension 2000; 36: 929 933.

5. Petar, N., Julia, N., Tanya, D., Fedya, N., Changes in intima media thickness, ankle brachial index and flow mediated dilation in pre hypertensive individuals. Scripta scientifica medica. 2015; 47(3): 15-20.

6. Chobanian, A. V., Bakris, G. L., Black, H. R., Cushman, W. C., Green, L. A., Izzo, J. L., et al., The seventh report of the joint national committee on prevention, detection, evaluation, and treatment of high blood pressure: the JNC 7 report. JAMA. 2003; 289(19): 2560-2572.

7. Parthaje, P. M., Unnikrishnan, B., Thankappan, K. R., Thapar, R., Fatt, Q. K., Oldenburg, B. Prevalence and correlates of prehypertension among adults in Urban South India. Asia Pac J Public Health. 2016; 28(1 Suppl): 93S$101 \mathrm{~S}$.

8. Mini, G. K., Sarma, P. S., Thankappan, K. R., Risk of progression to hypertension from prehypertension and normal blood pressure: Results from a prospective cohort study among industrial workers in Kerela, India. Heart Mind 2018; 2: 106-110.

9. Han, M., Li, Q., Liu, L., Zhang, D., Ren, Y., Zhao, Y., et al., Prehypertension and risk of cardiovascular diseases: a metaanalysis of 47 cohort studies. J Hypertens. 2019; 37(12): 2325-2332.

10. Mainous, A. G., Everett, C. J., Liszka, H., King, D. E., Egan, B. M., Prehypertension and mortality in a nationally representative cohort. Am J Cardiol. 2004; 94(12): 14961500 .

11. Urbina, E. M., Khoury, P. R., McCoy, C., Daniels, S. R., Kimball, T. R., Dolan, L. M. Cardiac and vascular consequences of prehypertension in youth. J Clin Hypertens. 2011; 13: 332-342.

12. Kim, S. H., Cho, G. Y., Baik, I., Lim, S. Y., Choi, C. U., Lim, H. E., et al., Early abnormalities of cardiovascular structure and function in middle aged Korean adults with prehypertension: The Korean Genome Epidemiology Study. Am J Hypertens. 2011; 24: 218-224.

13. Liu, B., Ni, J., Shi, M., Zhan, C., Lu, H., Wu, Y., et al., Carotid intima-media thickness and its association with conventional risk factors in low-income adults: a populationbased cross-sectional study in China. Sci Rep. 2017; 7: 41500 .

14. Jacoby, D. S., Mohler, I. E., Rader, D. J., Noninvasive atherosclerosis imaging for predicting cardiovascular events and assessing therapeutic interventions. Curr Atheroscler Rep. 2004; 6: 20-26.

15. Hansa, G., Bhargava, K., Bansal, M., Tandon, S., Kasliwal, R. R., Carotid intima media thickness and coronary artery disease: An Indian perspective. Asian Cardiovasc Thorac Ann. 2003; 11: 217-221.

16. Whelton, P. K., Carey, R. M., Aronow, W. S., Casey, D. E., Collins, K. J., Himmelfarb, C. D., et al., 2017 ACC/AHA Guideline for the Prevention, Detection, Evaluation, and Management of High Blood Pressure in Adults: A Report of the American College of Cardiology/American Heart Association Task Force on Clinical Practice Guidelines. J Am Coll Cardiol. 2018; 71: e127-e248.

17. Zhang, M., Batu, B., Tong, W., Li, H., Lin, Z., Li, Y., et al., Prehypertension and cardiovascular risk factor clustering among Mongolian population in rural and animal husbandry area, Inner Mongolia, China. Circ J. 2009; 73: 1437-1441.

18. Lankarani, K. B., Ghaffarpasand, F., Mahmoodi, M., Dehghankhalili, M., Honarvar, B., Lotfi, M., et al., Predictors of common carotid artery intima-media thickness and atherosclerosis in a sample of Iranian general population. Shiraz E-Med J. 2015; 16(5): e27906.

19. Yadav, S., Boddula, R., Genitta, G., Bhatia, V., Bansal, B., Kongara, S., et al., Prevalence and risk factors of pre- hypertension and hypertension in an affluent north Indian population. Indian J Med Res. 2008; 128: 712-720.

20. Mohan, V., Deepa, M., Farooq, S., Datta, M., Deepa, R., Prevalence, awareness and control of hypertension in Chennai-The Chennai Urban Rural Epidemiology Study (CURES-52). J Assoc Physicians India. 2007; 55: 326-332.

21. Cuspidi, C., Sala, C., Tadic, M., Gherbesi, E., Grassi, G., Mancia, G. Pre-hypertension and subclinical cardiac damage: A meta-analysis of echocardiographic studies. Int J Cardiol. 2018; 270: 302-308.

22. De Marco, M., de Simone, G., Roman, M.J., Chinali, M., Lee, E. T., Russell, M., et al., Cardiovascular and metabolic predictors of progression of prehypertension into hypertension: the Strong Heart Study. Hypertension 2009; 54(5): 974-980.

23. Salonen, J. T., Salonen, R. Ultrasound B-mode imaging in observational studies of atherosclerotic progression. Circulation. 1993; 87: II56-65. 